\title{
Rapid and sustained response to immune checkpoint inhibition in cutaneous squamous cell carcinoma after allogenic hematopoietic cell transplant for sézary syndrome
}

\author{
Karam Khaddour ${ }^{1,2}$ (D) Amy Musiek ${ }^{3}$, Lynn A. Cornelius ${ }^{3,4}$, Farrokh Dehdashti ${ }^{5,4}$, Peter Westervelt ${ }^{2,4}$,
} Ryan Fields $s^{6,4}$ and George Ansstas ${ }^{1,4^{*}}$

\begin{abstract}
Background: Cutaneous squamous cell carcinoma (CSCC) is not uncommon in association with indolent malignancies that were treated with prior radiotherapy and after allogenic bone marrow transplantation. On the other hand, cutaneous T-cell lymphoma (CTCL) is a subtype of non-Hodgkin's lymphoma which is characterized by an indolent course, with relative refractoriness to conventional chemotherapies and radiotherapy, and occasionally referred for allogeneic hematopoietic cell transplantation (allo-HCT). Recently, the use of immune checkpoint inhibitors has gained attention in the treatment of both cutaneous squamous cell carcinoma and hematological malignancies. However, many patients with hematological malignancies eventually undergo allo-HCT, raising the concern of potential adverse events (graft versus host disease) due to manipulation of the immune system with use of checkpoint inhibitors.

Case presentation: We describe a patient with relapsed refractory CTCL (Sézary Syndrome) who underwent allo-HCT with persistence of disease post-transplant. The patient additionally developed a progressively worsening lesion on the right shoulder which was biopsied and showed poorly differentiated carcinoma (CSCC).

Pembrolizumab was started for the treatment of CSCC. After second cycle of treatment, the CSCC lesion responded dramatically to the use of immune checkpoint inhibitor. Also, the patient experienced significant resolution of pruritus and generalized erythema. During 24 months of follow up after initial treatment with checkpoint inhibition immunotherapy, the patient showed durable response of both CSCC and CTCL, as well as restoration of full donor chimerism, without obvious worsening of graft versus host disease (GVHD).

Conclusion: This is the first case to our knowledge of rapid and durable response of both CSCC and CTCL to immune checkpoint inhibition after allo-HCT. Although this report highlights the potential for significant response to this class of medication, further studies are required to confirm the efficacy and safety of this approach in patients with $\mathrm{CTCL}$ after allo-HCT given the potential concern of GVHD.
\end{abstract}

Keywords: Cutaneous squamous cell carcinoma, Cutaneous T-cell lymphoma, Sézary syndrome, Immune checkpoint inhibitors, Allogenic hematopoietic cell transplant, Pembrolizumab, Graft versus host disease

\footnotetext{
*Correspondence: gasnsstas@wustl.edu

'Division of Medical Oncology, Department of Medicine, Washington

University in Saint Louis, 660 South Euclid Avenue, Campus Box 8056, St.

Louis, MO 63110, USA

${ }^{4}$ Alvin J. Siteman Cancer Center, St. Louis, USA

Full list of author information is available at the end of the article
}

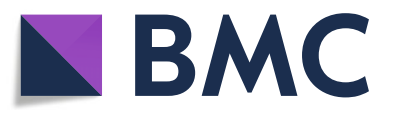

(c) The Author(s). 2019 Open Access This article is distributed under the terms of the Creative Commons Attribution 4.0 International License (http://creativecommons.org/licenses/by/4.0/), which permits unrestricted use, distribution, and reproduction in any medium, provided you give appropriate credit to the original author(s) and the source, provide a link to the Creative Commons license, and indicate if changes were made. The Creative Commons Public Domain Dedication waiver (http://creativecommons.org/publicdomain/zero/1.0/) applies to the data made available in this article, unless otherwise stated. 


\section{Background}

Conventional chemotherapies are considered not to be curative in the majority of cutaneous T-cell lymphomas (CTCL) [1]. Recently, the use of immune checkpoint inhibitors has largely expanded to include hematological malignancies, specifically relapsed/refractory chemoresistant Hodgkin's Lymphoma (r/r-cHL) and primary mediastinal large B-cell lymphoma [2, 3].The rationale relies on the fact that genetic alterations occurring in the microenvironment of lymphomas specifically in the programmed death ligand receptor (PD-L1/PD-L2) loci can lead to overexpression of PD-L1/2 on malignant cells, which helps tumor cells to evade the effective antitumor immune response [4]. Similarly, CTCL such as Mycosis Fungoides (MF) and Sézary Syndrome (SS) can debilitate the immune response against malignant cells in the tumor microenvironment and thus could be considered a target for therapies that can restore immune surveillance [5]. However, there is a concern for the use of immune checkpoint inhibitors in patients with lymphoma who undergo allogenic hematopoietic cell transplantation due to the possibility of triggering or aggravating graft versus host disease. This has led to lack of literature regarding the safety and efficacy of immune checkpoint inhibitors in this population as patients with prior history of allo-HCT were excluded from clinical trials which examined the efficacy of immunotherapy. Moreover, advanced cutaneous squamous cell carcinoma has been shown to have a high mutational burden which could increase the expression of tumor neoantigens [6]. Also, the association with PD-L1 expression is established with cutaneous squamous cell carcinoma which prompted the study of immune checkpoint inhibitors as a potential therapy [7].

We describe the first case to our knowledge of a patient with a history of allo-HCT who had a rapid and durable response of both $\mathrm{CSCC}$ and CTCL/SS after treatment with immune checkpoint inhibitors.

\section{Case presentation}

A 58-year-old Caucasian male with relapsed/refractory Sézary Syndrome (r/r SS) stage IVA was referred for allogeneic hematopoietic cell transplantation (alloHCT). The patient had received multiple therapies prior to being assessed for a transplant (Table 1). Positron Emission Tomography/Computed Tomography (PET/ $\mathrm{CT})$ with $\left[{ }^{18} \mathrm{~F}\right]$ fluorodeoxyglucose (FDG) prior to transplant (1/17/2017) revealed interval development of a new hypermetabolic soft tissue nodule in the skin of the posterior right shoulder with maximum standard uptake value $\left(\mathrm{SUV}_{\max }\right)$ of 12.3 and a hypermetabolic right paratracheal lymph node (Fig. 1). Skin biopsy was consistent with residual CTCL/SS, a bone marrow biopsy demonstrated hypocellularity with extensive involvement of
Table 1 Sequence of systemic therapies administered since diagnosis of cutaneous T-cell lymphoma/ Sézary Syndrome

\begin{tabular}{|c|c|c|}
\hline \multirow{2}{*}{$\begin{array}{l}\text { Prior therapy for CTCL/ } \\
\text { SS } \\
\text { Photochemotherapy } \\
\text { (PUVA) }\end{array}$} & \multicolumn{2}{|l|}{ Sequence and Duration } \\
\hline & First Line of Therapy & 8 months (2009) \\
\hline Photopheresis & $\begin{array}{l}\text { Second Line of } \\
\text { Therapy }\end{array}$ & 31 months (2009-2012) \\
\hline Romidepsin & Third Line of Therapy & 48 months (2010-2014) \\
\hline $\begin{array}{l}\text { Pegylated liposomal } \\
\text { doxorubicin }\end{array}$ & $\begin{array}{l}\text { Fourth Line of } \\
\text { Therapy }\end{array}$ & 3 months (2014) \\
\hline Gemcitabine & Fifth Line of Therapy & 1 month (2014) \\
\hline Alemtuzumab & Sixth Line of Therapy & 2 months (2015) \\
\hline Mogamulizumab & $\begin{array}{l}\text { Seventh Line of } \\
\text { Therapy }\end{array}$ & 1 month (2015) \\
\hline $\begin{array}{l}\text { Total skin electron } \\
\text { beam therapy }\end{array}$ & $\begin{array}{l}\text { Eighth Line of } \\
\text { Therapy }\end{array}$ & 3 months (2016) \\
\hline Bexarotene & Ninth Line of Therapy & 2 months (2016) \\
\hline Pralatrexate & $\begin{array}{l}\text { Tenth Line of } \\
\text { Therapy }\end{array}$ & 2 months (2016) \\
\hline $\begin{array}{l}\text { ICE (Ifosfamide, } \\
\text { Carboplatin, Etoposide) }\end{array}$ & $\begin{array}{l}\text { Eleventh Line of } \\
\text { Therapy }\end{array}$ & 3 months (2016-2017) \\
\hline $\begin{array}{l}\text { Sibling allogenic stem } \\
\text { cell transplantation }\end{array}$ & $\begin{array}{l}\text { Twelfth Line of } \\
\text { Therapy }\end{array}$ & $(2017)$ \\
\hline
\end{tabular}

mature T-cell lymphoma (79\% by flow cytometry), and cytogenetic studies revealed no aberrations. The patient underwent allogeneic hematopoietic cell transplantation (allo-HCT) using a fully matched male sibling donor. The myeloablative conditioning regimen prior to transplant consisted of hyperfractionated total body irradiation combined with high dose cyclophosphamide. Graft versus host disease (GVHD) prophylaxis consisted of tacrolimus and methotrexate. Tacrolimus was subsequently changed to sirolimus due to peri-transplant neurotoxicity. Shortly after transplantation (week 7), the patient developed a diffuse erythematous rash, a biopsy of which was most consistent with skin GVHD (vs CTCL vs drug rash) and received prednisone, mycophenolate mofetil (MMF) with ongoing sirolimus with improvement, and subsequent tapering to low dose prednisone ( $5 \mathrm{mg}$ daily/ week 23 ). A bone marrow biopsy 4 months after allo-HCT showed persistent marrow (and peripheral blood) involvement with sézary cells (15\% bone marrow involvement by mature $\mathrm{T}$-cell lymphoma with $\mathrm{CD} 4+/ \mathrm{CD} 8+$ ratio $>100$ ). There was also mixed chimerism by short tandem repeat (STR) assay with $82 \%$ donor cells (Fig. 2). There was incomplete resolution of the skin manifestations of CTCL, which included generalized erythema and pruritus. Repeated skin biopsies were consistent with residual CTCL/SS. Five months after allo-HCT, STR studies again demonstrated persistent mixed chimerism, and donor lymphocyte infusions (DLI) in two separate doses were administered on 


\section{a. $01 / 17 / 2017$}

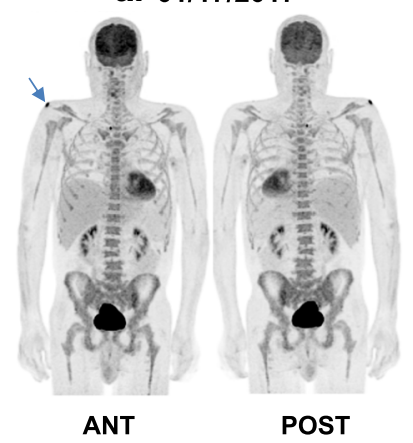

b. 09/05/2017

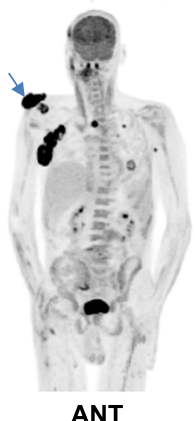

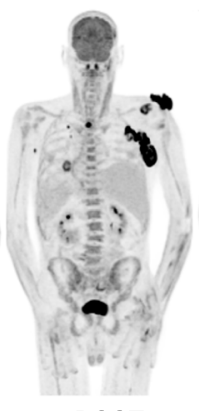

POST



ANT

\section{1/16/2018}



Fig. 1 Anterior and posterior volume-rendered maximum activity-reprojection FDG-PET images showing (a) FDG uptake in the right shoulder (arrow) and a paratracheal lymph node. b markedly hypermetabolic lesion in the right shoulder with SUV $\max$ of 26.7 (arrow), markedly hypermetabolic right axillary and right paratracheal lymphadenopathy with $S U V_{\max }$ of 27.3 with interval development of hypermetabolic focal cutaneous lesions, left axillary lymph node and pulmonary lesions. c near complete response of the right shoulder hypermetabolic lesion (arrow), complete resolution of paratracheal lymph node, left axillary lymph node, pulmonary and cutaneous lesions, with persistent FDG uptake of the right axillary lymph nodes

weeks 26 and 31 ( 1 and 3 X 10e7 CD3 cells/kg, respectively) without complete resolution of skin symptoms or erythema. Following allo-HCT, there was a gradual worsening of a right shoulder skin lesion noted on PET/ CT prior to transplant, and thought to be related to mycosis fungoides secondary to CTCL. The skin mass eventually extended over the superior aspect of the right shoulder with ulceration and hard induration, and 3 months post allo-HCT, the patient underwent subsequent involved-field radiotherapy with a total dose of 50 Gy. The lesion persisted and grew to $17 \times 10 \mathrm{~cm}$ in maximum dimension with worsening necrosis, ulceration, and spread to the anterior chest wall (Fig. 3), a skin biopsy was performed and histopathology revealed poorly differentiated squamous cell carcinoma. Magnetic Resonance Imaging (MRI) of the shoulder showed a right lesion with no bone or muscular invasion, but there was extensive right axillary lymphadenopathy. FDG-PET/CT revealed worsening of disease with extensive markedly hypermetabolic soft tissue thickening in the right shoulder with $\mathrm{SUV}_{\max }$ of 26.7 and interval development of markedly hypermetabolic partially necrotic right axillary lymphadenopathy with $\mathrm{SUV}_{\max }$ of 27.3 , as well as worsening in FDG uptake of the right paratracheal lymph

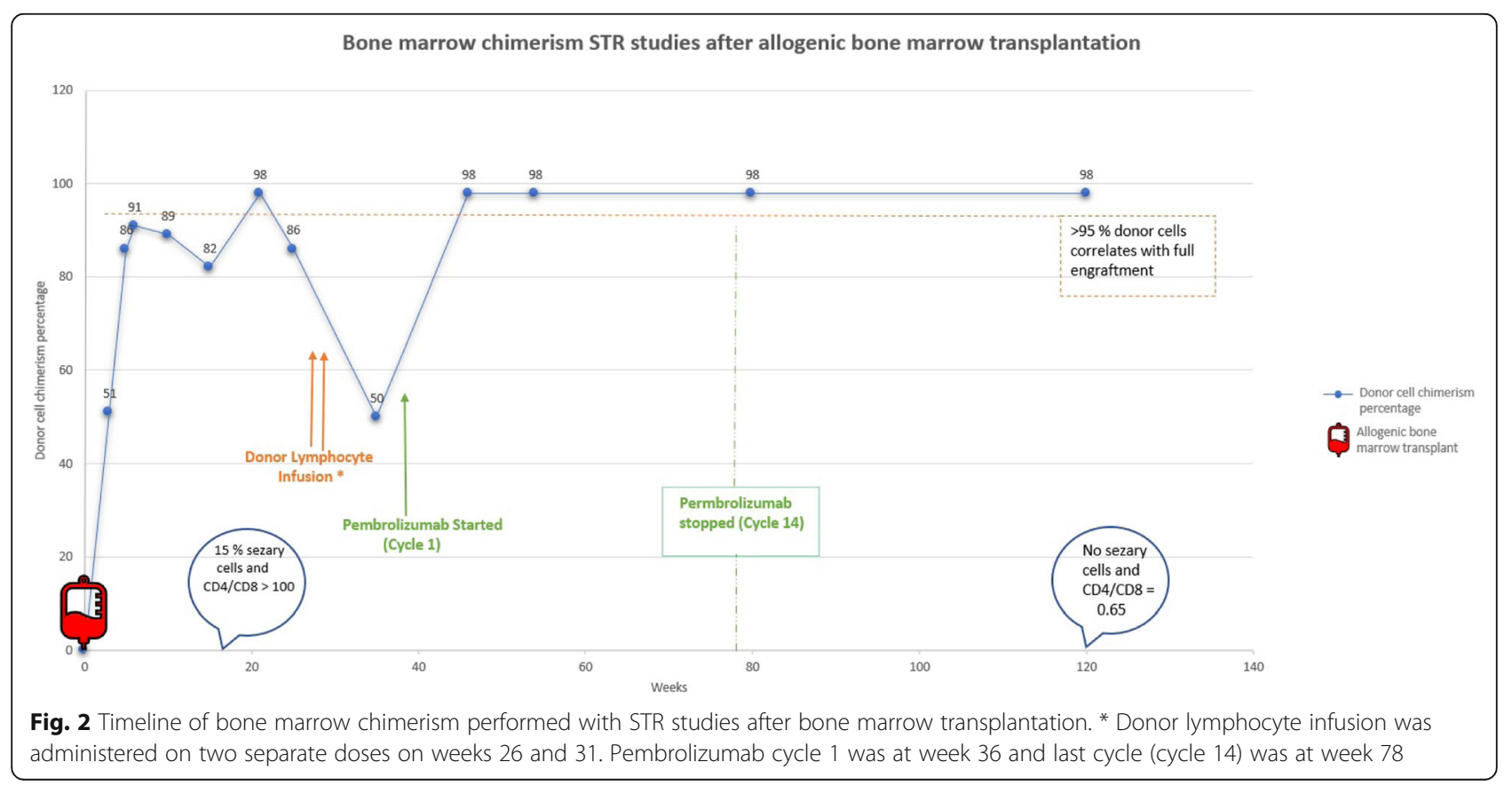



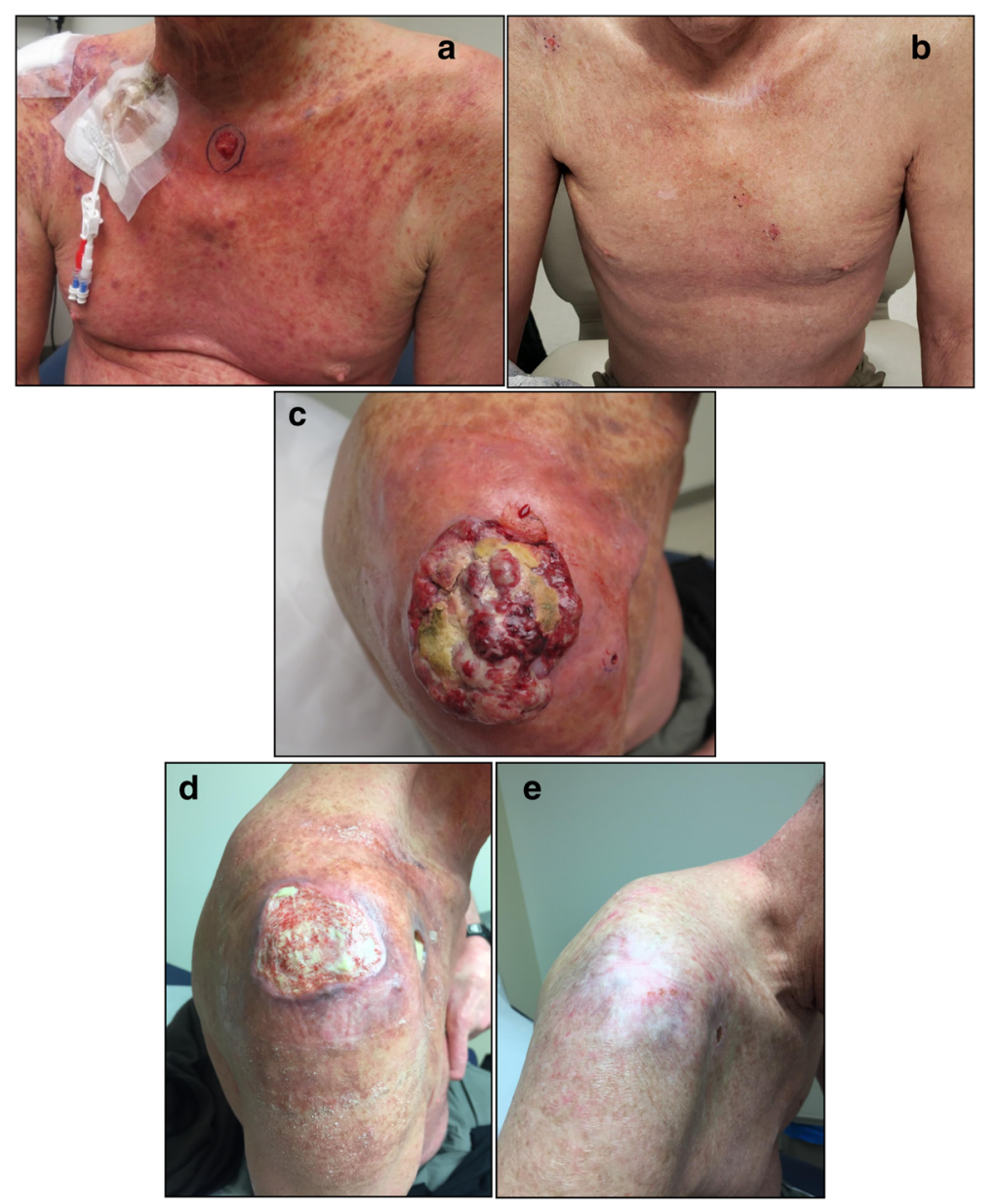

Fig. 3 Skin lesions of CTCL and CSCC before and after PD-1 inhibition. a: Generalized eruption of small confluent erythematous macules and papules on the anterior chest wall after stem cell transplant and before pembrolizumab. $\mathbf{b}$ : resolution of the previously mentioned erythematous maculopapular eruption after pembrolizumab. c: multiple firm nodules with partial ulceration and keratin deposition on the superior aspect of the right shoulder representing poorly differentiated squamous cell carcinoma (before pembrolizumab), also erythematous eruption can be noted around the CSCC lesion which represents cutaneous lymphoma. d \& e: Granular tissue with skin regeneration replacing the nodular ulcerated lesions of CSCC (after pembrolizumab), there is complete resolution of the papular erythematous rash. CTCL: cutaneous T cell lymphoma, cSCC: cutaneous squamous cell carcinoma

node. There was interval development of hypermetabolic focal cutaneous lesions, left axillary lymph nodes and pulmonary lesions (T3N2B; Stage IV SCC) (Fig. 1).

After discussion of treatment options with the patient, intravenous pembrolizumab was started at a dose of $200 \mathrm{mg}$ every 3 weeks. The patient had an Eastern Cooperative Oncology Group performance of (ECOG 3) prior to beginning of treatment. After $2^{\text {nd }}$ cycle of pembrolizumab, a macular rash appeared on the lower back. Skin biopsy demonstrated superficial perivascular mixed inflammatory cell infiltrate with normal $\mathrm{CD} 4+/ \mathrm{CD} 8+$ ratio and was consistent with grade I skin immune related adverse event (IRAEs) due to pembrolizumab. Prednisone $60 \mathrm{mg}$ was started and tapered over a total of 4 weeks with complete resolution of the skin symptoms secondary to IRAEs. The cutaneous manifestations including generalized erythema and pruritus which were secondary to CTCL resolved completely after the $2^{\text {nd }}$ cycle of immunotherapy. A FDG-PET/CT after the $5^{\text {th }}$ cycle of pembrolizumab showed marked metabolic response and near-complete resolution of the superficial right shoulder mass ( $\mathrm{SUV}_{\max }$ of 1.9), and complete resolution of right paratracheal and left axillary lymph nodes, as well as pulmonary and cutaneous lesions. There was decreased but persistent FDG uptake in the necrotic right axillary lymph nodes $\left(\mathrm{SUV}_{\max }\right.$ of 18.2) (Fig. 1). Given the rapid and significant response, pembrolizumab was continued for another three cycles and repeated PET/CT showed continued response with decreased FDG avidity in the right axilla. After cycle 14, the patient underwent right axillary lymph node dissection encompassing levels 1-3 nodes for assessment of the residual persistent lymphadenopathy on the right side. Histopathology demonstrated metastatic squamous cell 
carcinoma in 3 of 17 lymph nodes. His post-operative course was uncomplicated. The complete course of pembrolizumab treatment consisted of 14 cycles, over a course of 9 months. Follow up 24 months after initiation of immune checkpoint blockade therapy (14 months after stopping pembrolizumab) revealed no evidence of recurrence of cSCC. There was no compelling evidence of either progression of CTCL/SS or a flare in GVHD throughout his course of checkpoint inhibition therapy (most recent peripheral blood flow cytometry on week 120 showing no morphologic or immunophenotypic evidence of atypical lymphocytosis or sézary cells and $\mathrm{CD} 4+/ \mathrm{CD} 8+$ ration of 0.65 ).

\section{Discussion}

Systemic treatment of locally advanced and metastatic cutaneous squamous cell carcinoma has been limited with the exception of recent data supporting the potential role of immune checkpoint blockade specifically cemiplimab [8] among other ongoing trials with other PD-1 and PD-L1 inhibitors (Clinical Trials.gov. Identifiers: NCT02978625, NCT02964559, NCT03284424 and NCT03108131). Also, the data supporting the use of immune checkpoint inhibitors in the treatment of hematological malignancies other than classical Hodgkin's Lymphoma (cHL) or primary mediastinal large Bcell lymphoma is limited. A new incentive of implementing immune checkpoint blockade in the treatment of $\mathrm{T}$ cell lymphomas is supported by preclinical studies that showed programmed cell death ligand-1 (PD-L1) receptors to be expressed on malignant cells which contribute to suppression of host immunity against the malignant cells. This leads to overgrowth in T-cell clones derived from non-Hodgkin lymphoma [9]. Our case demonstrates a rapid and durable response after treatment with immune checkpoint inhibitors manifested by a resolution of CTCL/SS symptoms within 6 weeks and a remission period of 24 months since initiation of a PD-1 inhibitor. The indication to use pembrolizumab in our patient was for the treatment of poorly differentiated cutaneous squamous cell carcinoma. The theoretical possibility of a derived benefit against CTCL/SS was taken into consideration when starting treatment. Also, the possibility of exacerbating GVHD was also considered. Both poorly differentiated cutaneous squamous cell carcinoma (CSCC) and CTCL/SS responded rapidly within 6 weeks ( 2 cycles) after initiation of PD-1 inhibitor, without an evidence of GVHD flare.

Early phase clinical trials using the PD-1 inhibitor (nivolumab) in refractory/relapsed cutaneous T-cell lymphomas demonstrated different objective response rates (ORR) ranging from $15 \%$ in mycosis fungoides (MF) to $40 \%$ in peripheral T-cell lymphoma/Sézary Syndrome (SS) in phase I studies (number of evaluable patients $=18$ ) [10].
However, the previous study excluded patients with prior allogenic hematopoietic cell transplant. A different study using pembrolizumab demonstrated an ORR of $38 \%$ in 24 evaluable patients with MF/SS stages Ib-IV with the longest duration of response reported to be 46 weeks [11]. Interestingly, there were higher response rates in retrospective studies examining the role of PD-1 inhibition after allo-HCT compared to patients who received immunotherapy prior to transplant for $\mathrm{r} / \mathrm{r}-\mathrm{cHL}[12,13]$. These two previous retrospective studies showed ORR of 95 and 77\% with 1 -year progression free survival (PFS) of $85.2 \%$ compared to ORR of 75 and $87 \%$ respectively in patients who received immune checkpoint inhibitors prior to transplant $[2,12-14]$. This raises the hypothesis that immune checkpoint blockade could have a synergistic role after allo-HCT or a role in homing of donor T-cells leading to an enhanced graft versus tumor effect. Some data support the previous hypothesis as immune escape is one of the mechanisms considered to cause relapse after transplant in hematological malignancies, and T-cell exhaustion is a potential mechanism of relapse after allo-HCT due to overexpression of $\mathrm{PD}-1 / \mathrm{PD}-\mathrm{L} 1$ receptors, which leads to inactivation of effective $\mathrm{T}$-cells in the tumor microenvironment [15]. Norde et al. found that relapsed myeloid leukemia after allo-HCT had higher expression and upregulation of PD-L1 receptors on progenitor malignant clones. It was also observed in the same study the suppressive effect on allogenic CD3+ T-cells when there was high expression of PD-L1 on malignant leukemic cells. Interestingly, blockade of the PD-1/PD-L1 interaction can augment the expansion of effector CD8+ Tcells and reactivate unresponsive T-memory cells in the microenvironment required for graft versus leukemia effect [15]. Moreover, there is a small body of evidence supporting a role of immune checkpoint inhibitors in the bone marrow microenvironment in acute myeloid leukemia (AML), myelodysplastic syndrome (MDS) and myelopthisis due to melanoma $[16,17]$.

The concern with the use of immune checkpoint inhibitors after allo-HCT is the development of graft versus host disease (GVHD). Conflicting data exist regarding the occurrence of this adverse event, with some studies reporting increased incidence and worsening of preexisting GVHD with $30 \%$ ( 6 of 20 patients), and $26 \%$ deaths in patients who received immune checkpoint blockade after allo-HCT $[12,13]$. In the previous two retrospective studies there were 10 deaths related to GVHD in 51 patients (23 of whom developed GVHD) $[12,13]$. However, another retrospective study assessing patients who were treated with immune checkpoint inhibitors after allo-HCT did not report GVHD development in any of the 7 evaluable patients [18].

The largest systematic review assessing the risk of GVHD with immune checkpoint inhibitors after alloHCT showed that $49 \%$ of patients who developed 
GVHD had a prior history of the disease [19]. Some of the predictive factors of GVHD development in this patient population were higher doses of immune checkpoint inhibitors, shorter intervals between transplant and start of immunotherapy, and previous history of GVHD. This systematic review also supports other observations of higher response rates when immune checkpoint inhibitors were administered after transplant compared to those who received immunotherapy before they underwent allo-HCT [12, 13, 19].

In our case, we did not observe GVHD after treatment with PD-1 inhibitors. In fact, the patient's erythema and pruritus which were manifestations of patient's CTCL improved significantly after starting pembrolizumab. Moreover, there was a dermatological immune related adverse event observed in the patient during treatment (after 2nd cycle of pembrolizumab) which correlated with the response of both CSCC and CTCL. The previous observation is supported by a retrospective study showing robust immune response to PD-1/PD-L1 inhibitors when lichenoid and spongiotic IRAEs develop during treatment with PD-1/PD-L1 inhibitors [20].

It should be noted that donor lymphocyte infusion (DLI) which was performed in our patient on weeks 26 and 31 could have potentially led to full engraftment of allo-HCT resulting in the response observed in CTCL. The median response from administration of DLI to full engraftment is estimated to be $8-12$ weeks [21]. However, the response of CSCC and CTCL in our case was observed to occur only after the administration of pembrolizumab (first cycle was at week 36) and the substantial deep response was observed after the second cycle which was at week 39 .

In conclusion, our case is the first to describe a rapid and sustained clinical response of both CTCL/SS and cSCC to immune checkpoint inhibition after allo-HCT, without development of GVHD. However, this observation should be interpreted with caution given the nontrivial concern of GVHD. Further larger studies are needed to confirm the efficacy of immune checkpoint inhibitors and their safety profile in this patient population.

\section{Abbreviations \\ Allo-HCT: allogenic hematopoietic stem cell transplant; CTCL: Cutaneous T cell Iymphoma; GVHD: Graft versus host disease; IRAEs: Immune related adverse events; PD-1: Programmed death receptor 1; PET/CT: Positron emission tomography- computed tomography; r/r-cHL: Relapsed/refractory classical Hodgkin's lymphoma; cSCC: Cutaneous squamous cell carcinoma; SS: Sézary syndrome}

\section{Acknowledgments}

Not applicable.

\section{Authors' contributions}

K.K performed the chart, literature review to support conclusions of this report, prepared Fig. 2 and wrote the manuscript with consultation with the other authors. F.D prepared Fig. 1 with supervision on the manuscript. R.C.F operated on the case and supervised the surgical peri-operative course. L.A.C \& A.M prepared Fig. 3 and supervised the work. G.A \& P.W supervised the entire work. All authors contributed to the final version of the manuscript. All authors read and approved the final manuscript.

\section{Funding}

No funding provided for this study.

\section{Availability of data and materials}

Data was acquired through patient chart review using Washington University in Saint Louis medical records.

Ethics approval and consent to participate Not Applicable.

\section{Consent for publication}

Written and verbal consent were obtained from the patient prior to submission for publication. A copy of the written consent form is available at the editorial office for this journal.

\section{Competing interests}

KK reports no conflict of interest. AM reports: kyowa kirin- advisory board, Helsinn- Advisory board, Elorac, Sologenix MiRagen (investigator), investigator in CITN study "embrolizymab and IFN-gamma in MF/SS and synovial sarcoma". LC reports no conflict of interest. FD reports no conflict of interest. PW reports no conflict of interest. RF reports no conflict of interest. GA speaker bureau for Merck.

\section{Author details}

${ }^{1}$ Division of Medical Oncology, Department of Medicine, Washington University in Saint Louis, 660 South Euclid Avenue, Campus Box 8056, St. Louis, MO 63110, USA. ${ }^{2}$ Division of Medical Oncology, Bone Marrow Transplantation and Leukemia, Department of Medicine, Washington University in Saint Louis, St. Louis, USA. ${ }^{3}$ Division of Dermatology, Department of Medicine, Washington University in Saint Louis, St. Louis, USA. ${ }^{4}$ Alvin J. Siteman Cancer Center, St. Louis, USA. ${ }^{5}$ Division of Nuclear Medicine, Department of Radiology, Washington University in Saint Louis, St. Louis, USA. ${ }^{6}$ Section of Surgical Oncology, Department of Surgery, Washington University in Saint Louis, St. Louis, USA.

Received: 23 August 2019 Accepted: 30 October 2019

Published online: 04 December 2019

\section{References}

1. Hughes CF, Khot A, McCormack C, Lade S, Westerman DA, Twigger R, Buelens $\mathrm{O}$, et al. Lack of durable disease control with chemotherapy for mycosis fungoides and Sezary syndrome: a comparative study of systemic therapy. Blood. 2015;125:71-81.

2. Chen $\mathrm{R}$, Zinzani $\mathrm{PL}$, et al. Phase II study of the efficacy and safety of Pembrolizumab for relapsed/refractory classic Hodgkin lymphoma. J Clin Oncol. 2017:35(19):2125-32.

3. Zinzani PL, Ribrag V, et al. Safety and tolerability of pembrolizumab in patients with relapsed/refractory primary mediastinal large B-cell lymphoma. Blood. 2017;130(3):267-70.

4. Roemer MG, Advani RH, Ligon AH, et al. PD-L1 and PD-L2 genetic alterations define classical Hodgkin lymphoma and predict outcome. J Clin Oncol. 2016:34(23):2690-7.

5. Sivanand A, Surmanowicz P, Alhusayen R, Hull P, Litvinov IV, Zhou Y, Gniadecki R. Immunotherapy for Cutaneous T-Cell Lymphoma: Current Landscape and Future Developments. J Cutan Med Surg. 2019;23(5):537-44.

6. Pickering $C R$, et al. Mutational landscape of aggressive cutaneous squamous cell carcinoma. Clin Cancer Res. 2014;20(24):6582-92.

7. Slater NA, et al. PD-L1 expression in cutaneous squamous cell carcinoma correlates with risk of metastasis. J Cutan Pathol. 2016:43(8):663-70.

8. Migden MR, et al. PD-1 blockade with Cemiplimab in advanced cutaneous squamous-cell carcinoma. N Engl J Med. 2018:379(4):341-51.

9. Wilcox RA, Feldman AL, et al. B7-H1 (PD-L1, CD274) suppresses host immunity in T-cell lymphoproliferative disorders. Blood. 2009;114(10): 2149-58. 
10. Lesokhin AM, Ansell SM, Armand P, et al. Nivolumab in patients with relapsed or refractory hematologic malignancy: preliminary results of a phase Ib study. J Clin Oncol. 2016;34(23):2698-704.

11. Khodadoust M, Rook AH, Porcu P, et al. Pembrolizumab for treatment of relapsed/refractory mycosis Fungoides and Sezary syndrome: clinical efficacy in a Citn multicenter phase 2 study. Blood. 2016;128.

12. Herbaux C, Gauthier J, Brice P, et al. Efficacy and tolerability of nivolumab after allogeneic transplantation for relapsed Hodgkin lymphoma. Blood. 2017;129(18):2471-8

13. Haverkos BM, Abbott D, Hamadani M, et al. PD-1 blockade for relapsed lymphoma postallogeneic hematopoietic cell transplant: high response rate but frequent GVHD. Blood. 2017;130(2):221-8.

14. Ansell SM, Lesokhin AM, Borrello I, et al. PD-1 blockade with nivolumab in relapsed or refractory Hodgkin's lymphoma. N Engl J Med. 2015;372(4):311-9.

15. Norde WJ, Maas F, et al. PD-1/PD-L1 interaction contribute to functional Tcell impairment in patients who relapse with cancer after allogenic stem cell transplantation. Cancer Res. 2011;71(15):5111-22.

16. Boddu P, Kantarjian $\mathrm{H}$, et al. The emerging role of immune checkpoint based approaches in AML and MDS. Leuk Lymphoma. 2018;59(4):790-802.

17. Rosner S, Sen F, Postow M. Response after treatment with pembrolizumab in a patient with myelophthisis due to melanoma: the role of checkpoint inhibition in the bone. J Immunother Cancer. 2017:5:34

18. Schoch LK, Borrello I, Fuchs EJ, et al. Checkpoint inhibitor therapy and graft versus host disease in allogeneic bone marrow transplant recipients of haploidentical and matched products with post-transplant cyclophosphamide [abstract]. Blood. 2016;128(22):Abstract 4571.

19. Ijaz A, Khan AY, Malik SU, et al. Significant risk of graft-versus-host disease with exposure to checkpoint inhibitors before and after allogeneic transplantation. Biol Blood Marrow Transplant. 2019;25(1):94-9.

20. Min Lee CK, Li S, Tran DC, Zhu GA, Kim J, Kwong BY, Chang ALS. Characterization of dermatitis after PD-1/PD-L1 inhibitor therapy and association with multiple oncologic outcomes: a retrospective case-control study. J Am Acad Dermatol. 2018:79(6):1047-52.

21. Deol A, Lum LG. Role of donor lymphocyte infusions in relapsed hematological malignancies after stem cell transplantation revisited. Cancer Treat Rev. 2010;36(7):528-38.

\section{Publisher's Note}

Springer Nature remains neutral with regard to jurisdictional claims in published maps and institutional affiliations.

Ready to submit your research? Choose BMC and benefit from:

- fast, convenient online submission

- thorough peer review by experienced researchers in your field

- rapid publication on acceptance

- support for research data, including large and complex data types

- gold Open Access which fosters wider collaboration and increased citations

- maximum visibility for your research: over $100 \mathrm{M}$ website views per year

At $\mathrm{BMC}$, research is always in progress.

Learn more biomedcentral.com/submissions 